\title{
Caracterización ecológica de una turbera ombrogénica en Magallanes: hacia una propuesta de bioindicadores de monitoreo ambiental
}

\author{
Ecological characterization of an ombrogenic peatland in Magallanes: towards a proposal of \\ bioindicators for environmental monitoring
}

\section{Erika Monsalve ${ }^{1}$, René Muñoz-Arriagada ${ }^{1}$, Nelson Bahamonde ${ }^{2} \&$ Osvaldo J. Vidal ${ }^{3, *}$}

'Departamento de Ciencias Agropecuarias y Acuícolas, Facultad de Ciencias, Universidad de Magallanes, Punta Arenas, Chile.

${ }^{2}$ ONG AMA Torres del Paine, Torres del Paine, Chile.

${ }^{3}$ Laboratorio de Botánica, Instituto de la Patagonia, Universidad de Magallanes, Punta Arenas, Chile.

*E-mail: osvaldo.vidal@umag.cl

\section{RESUMEN}

Las turberas ombrogénicas son ecosistemas ecológicamente extremos, donde las condiciones restrictivas como anoxia, alta acidez y baja disponibilidad de nutrientes permiten la acumulación paulatina del sustrato orgánico conocido como turba. Este sustrato es de gran interés comercial pues es explotado para fines horticulturales y biomasa energética. La explotación de turba, sin embargo, implica la remoción de la vegetación, lo que supone un efecto importante para el restablecimiento de la sucesión secundaria y resiliencia ecosistémica. Se estudió la flora y la vegetación de turberas en Península de Brunswick (Magallanes), en un área susceptible de explotación minera, para caracterizar indicadores ecológicos. Se realizaron censos fitosociológicos para determinar atributos de composición y estructura a escala de comunidad y paisaje. Esta información fue cotejada con imágenes satelitales y analizada mediante procedimientos univariados (e.g. K-W, Pearson) y multivariados (e.g. NMDS, ISA, ANOSIM). Se diferenciaron los siguientes tipos de vegetación en turberas ombrogénicas: turbera graminoide, camefítica, esfagnosa y pulvinada, las cuales se asociaron a un gradiente de terrestrialización a paludificación, y de descomposición. La mayor riqueza promedio se registró en las turberas pulvinadas. Las turberas camefíticas y graminoides registraron las mayores superficies, mientras que las pulvinadas se distribuyeron en pocas hectáreas. Se registraron especies diagnósticas (e.g. Caltha appendiculata, Donatia fascicularis, Drosera uniflora, Myrteola nummularia) asociadas a uno u otro tipo de vegetación, denotando preferencias ecológicas. No se registraron especies exóticas. Esta información puede ayudar a definir niveles críticos de resiliencia y proponer estrategias de manejo y restauración en turberas abandonadas post-perturbación.

Palabras clave: descomposición von Post, indicadores de biodiversidad, Península de Brunswick, Sphagnum magellanicum, turberas prístinas.

\section{ABSTRACT}

Ombrogenic peat bogs are ecologically extreme ecosystems, where the restrictive conditions such as anoxia, high acidity and low availability of nutrients allow a gradual accumulation of the organic substrate known as peat. This substrate is of great commercial interest because it is exploited for horticultural purposes and energy biomass. The exploitation of peat, however, involves the removal of vegetation, which has an important effect on secondary succession and ecosystem resilience. The flora and vegetation 
of peat bogs in the Brunswick Peninsula (Magallanes), in an area susceptible to mining exploitation, was studied to characterize ecological indicators. Phytosociological surveys were carried out to determine attributes of composition and structure at the community and landscape scale. This information was collated with satellite images and analyzed by univariate procedures (e.g. KW, Pearson) and multivariate (e.g. NMDS, ISA, ANOSIM). The following types of ombrogenic peat bogs were differentiated: graminoid, chamaephytic, Sphagnum and pulvinated peat bogs, which were associated with a terrestrialization to paludification gradient, and decomposition. The highest average richness was recorded in the pulvinated peat bogs. The chamaephytic and graminoid bogs has the largest areas, while the pulvinated were distributed in a few hectares. Diagnostic species (e.g. Caltha appendiculata, Donatia fascicularis, Drosera uniflora, Myrteola nummularia) were associated with one or another type of vegetation, showing ecological preferences. No exotic species were recorded. This information can help define critical levels of resilience and propose management and restoration strategies in post-disturbance abandoned peatlands.

Keywords: biodiversity indicators, Brunswick peninsula, pristine peats, Sphagnum magellanicum, von Post decomposition.

\section{INTRODUCCIÓN}

Las turberas del sur de Chile y Argentina son humedales localizados en áreas templadas frías y húmedas, donde condiciones ecológicas restrictivas como la continua saturación de agua, baja disponibilidad de nutrientes, alta acidez y anoxia, propician niveles bajos de descomposición de materia orgánica y así, ocurre una lenta pero eficiente acumulación de sustrato orgánico, conocido como turba (Ramírez \& San Martín 2008, Domínguez \& Vega-Valdéz 2015). Desde el punto de vista florístico, fisionómico y/o ecológico, las turberas han sido clasificadas, entre muchas, como turberas esfagnosas, turberas pulvinadas, turberas de tundra montana y turberas cipero-graminoides, las que varían dependiendo de factores ambientales como la profundidad de la napa freática, el grado de descomposición de la turba, el gradiente de acidez-alcalinidad, el relieve y la continentalidad, entre otros (Pisano 1977, Kleinebecker et al. 2007, Ramírez \& San Martín 2008, Amigo et al. 2017). En Chile estos ecosistemas se distribuyen desde la Región de Los Ríos (latitud $40^{\circ} \mathrm{S}$ ) hasta Magallanes (Ramírez \& San Martín 2008), cubriendo una superficie estimada total de 7.684.000 ha (Luebert \& Pliscoff 2006), de las cuales cerca de 2.270 .000 ha $(29,5 \%)$ se encuentran en Magallanes. La mayor parte de esta última superficie $(83 \%)$ se ubica dentro de las áreas administradas por el Sistema Nacional de Áreas Silvestres Protegidas del Estado (SNASPE), lo que supone un alto grado de conservación y protección de estos sensibles e importantes ecosistemas (Ruiz \& Doberti 2005). En las turberas esfagnosas, la especie dominante, el musgo Sphagnum magellanicum Brid. adquiere una fuerte ventaja competitiva debido, entre otros factores, a la alta capilaridad de sus tejidos, alta productividad bajo condiciones pobres de nutrientes, contenidos altos de ácido urónico y tejido muerto recalcitrante (Van Breemen 1995).

Las turberas son ecosistemas que albergan una flora y fauna única y especializada, y representan una alta significancia ecológica debido a los servicios y bienes ecosistémicos que pueden proporcionar y que incluyen, entre otros, almacenamiento de carbono, regulación del clima, registros paleoclimáticos, provisión de agua, provisión de fibra, combustible y recreación (Joosten \& Clarke 2002, Kimmel \& Mander 2010, Domínguez \& Vega-Valdéz 2015). Durante los últimos años, se ha registrado una creciente demanda para el uso económico de los dos principales bienes que puede aportar este ecosistema. Por una parte, el musgo Sphagnum es utilizado como sustrato natural en horticultura, embalaje para el transporte de bulbos y flores y para el establecimiento de frutales y especies forestales (Tapia 2008), entre otros usos. Por otra parte, la turba es usada dependiendo de su grado de descomposición (i.e. color): la turba rubia, con menor grado de descomposición (i.e. von Post 1-3), es usada para fines horticulturales y tratamiento de aguas residuales, mientras que la turba negra, con mayores niveles de descomposición (i.e von Post $>6$ ), es usada como combustible. Un grado intermedio de descomposición de la turba (i.e. von Post 4-5) es usada para la mejora de suelos (Ruiz \& Doberti 2005). La demanda de la turba ha incrementado desde 768 toneladas hasta 3.621 toneladas desde el 2009 al 2016, respectivamente, con un aumento del $57 \%$ entre los años 2015 y 2016 (SERNAGEOMIN 2018). 
La extracción del recurso Sphagnum y la turba en Chile sucede de manera separada mediante dos procedimientos diferentes exigidos por la legislación Chilena (Decreto 25, MINAGRI 2018). El musgo Sphagnum requiere la corta manual o con herramientas no intrusivas (sin maquinaria), sin drenaje del suelo y permitiendo un remanente de cobertura del 30\%. Dependiendo del sitio de cosecha, los períodos de rotación deben ser de 12 años (regiones de Los Ríos y Los Lagos) a 85 años (regiones de Aysén y Magallanes). Por otra parte, la extracción de la turba ocurre generalmente mediante la confección de zanjas de drenaje de los sitios a intervenir, a lo que prosigue la extracción de turba en bloques con uso de maquinaria. Consecuentemente, los sitios intervenidos se constituyen de trincheras explotadas y fajas paralelas donde se apila los bloques de turba para su secado y transporte (Domínguez et al. 2012, Domínguez 2014). Sea que se trate de explotación del musgo Sphagnum o de extracción de turba, los sitios intervenidos son abandonados post-explotación, lo que permitiría el restablecimiento de la vegetación vía sucesión secundaria o restauración pasiva. Sin embargo, hasta el momento existen muy pocos estudios que permitan determinar las vías sucesionales post-perturbación. Para ello, se requiere generar criterios e indicadores de biodiversidad (sensu Noss 1990), que puedan ser monitoreados con fines de restauración ecológica activa y/o pasiva, o monitoreo ambiental (SER 2004). Domínguez et al. (2012), por ejemplo, compararon la vegetación de sitios perturbados por extracción minera de turba, con sitios no explotados en una turbera de río Rubens en la provincia de Última Esperanza (Magallanes). Sus resultados mostraron un incipiente proceso de invasión biológica promovido por especies como Holcus lanatus L., Rumex acetosella L., Carex canescens L. y Hieracium pilosella L. (Domínguez et al. 2012). Estas especies representaron el $11,15 \%$ del ensamble 20 años post-perturbación, denotando un efecto a largo plazo de la explotación. De esta manera, la sucesión secundaria tendría el potencial de generar un cambio de estado hacia ecosistemas diferentes del original.

El sitio "La Grazzia", localizado al sur de la Península de Brunswick (Magallanes), corresponde a un área de 279 ha asignada a extracción minera, donde la extracción de turba se realiza mediante explotación de trincheras, intercaladas con áreas no explotadas. Los "paños" bajo explotación contienen zanjas de drenaje y la explotación se realiza con la ayuda de maquinaria pesada. Adyacente a los paños de explotación, se encuentra un área no intervenida de 314 ha. Esta situación representa una interesante posibilidad para generar un estudio de caso y evaluar las turberas no intervenidas para, consecuentemente, proponer indicadores de biodiversidad factibles de ser evaluados con fines de restauración ecológica y/o monitoreo ambiental. Esta caracterización ecológica es muy importante, debido a la significancia que representa este tipo de paisaje frente a problemas recientes como calentamiento global y demanda incrementada del recurso turba. Además, al igual que en otros ecosistemas en Chile, se carece de directrices e indicadores ecológicos que permitan evaluar el efecto de actividades extractivas intensivas o extensivas. A través de este estudio, nos proponemos el siguiente objetivo general: Proponer indicadores de biodiversidad en turberas ombrogénicas de la Península de Brunswick (Magallanes), como información base para acciones de restauración ecológica y/o monitoreo ambiental. Nuestros objetivos específicos son: 1) Caracterizar la flora y vegetación de los diferentes tipos de turberas del sitio La Grazzia; 2) Relacionar la vegetación de turberas ombrogénicas con principales gradientes ecológicos y 3) Comparar atributos de composición y estructura para la vegetación del sitio de estudio.

\section{MATERIALES Y MÉTODOS}

\section{DESCRIPCIÓN DEL ÁREA DE ESTUDIO}

El área de estudio se denomina sector "La Grazzia" (370142 mE - 4052136 mS) y se ubica en la Península de Brunswick, en la Región de Magallanes y Antártica Chilena. El sitio está distante 61 kilómetros al sur de la ciudad de Punta Arenas. El clima puede ser clasificado como trasandino con degeneración esteparia $(E T)$, con precipitaciones anuales que fluctúan entre 400 a $620 \mathrm{~mm}$ (Pisano 1977). El substrato geológico se compone por formaciones antiguas, típicamente rocas metamórficas del precámbricoy/o paleozoico, con relieves más o menos suavizados por depósitos coluviales y glaciofluviales (Pisano 1973). Los suelos son de tipo Bog originados a partir de turberas ombrogénicas, formados principalmente por el musgo Sphagnum magellanicum que puede estar parcial o totalmente descompuesto. Los valores de $\mathrm{pH}$ en este tipo de turberas son fuertemente ácidos, con valores fluctuando entre 3,2 y 4,0 (Pisano 1973). La vegetación en esta turbera ombrogénica está dominada por el musgo Sphagnum magellanicum, que forma una cubierta ininterrumpida, excepto por pozos y pequeños cursos de escurrimiento de escasa profundidad y fondos fangosos. El relieve en el sitio de estudio es elevado y convexo, dependiendo de las condiciones abióticas que propician el crecimiento diferencial del musgo Sphagnum. Junto a esta especie y conformando mosaicos de variada superficie, codominan otras especies características de turberas ombrogénicas, como la murtilla (Empetrum rubrum Vahl ex Willd.), la chaura enana (Gaultheria pumila (L.f.) D.J. Middleton), el tetroncio (Tetroncium magellanicum Willd.), el junquillo (Marsippospermum grandiflorum (L.f.) Hook. f.) 
y el Ñirre (Nothofagus antarctica (G.Forst.) Oerst.). Entre otras especies de menor frecuencia en el área de estudio, pero con dominancia local, pueden mencionarse la donatia (Donatia fascicularis J.R. Forst. \& G. Forst.), la calta (Caltha appendiculata Pers.), la drosera (Drosera uniflora Willd.) y la mirteola (Myrteola nummularia (Poir.) O. Berg) (Pisano 1977, Domínguez et al. 2012).

\section{Perturbaciones antrópicas en el Área de ESTUdio}

La superficie que comprende las turberas ombrogénicas en el área de estudio abarca 608 ha, distribuidas en 14 fragmentos flanqueados por franjas boscosas en las zonas de drenaje intermedio (Pisano, 1973). En estos márgenes se desarrollan especies arbóreas como el Nirre (Nothofagus antarctica (G.Forst.) Oerst.), el coihue de Magallanes (Nothofagus betuloides (Mirb.) Oerst.) y el canelo (Drimys winteri J.R. Forst. \& G. Forst.), junto con especies de sotobosque como el romerillo (Chiliotrichum diffusum (G.Forst.) Kuntze), el michay (Berberis ilicifolia L.f.) y herbáceas como el pasto azul (Alopecurus magellanicus Lam.) y el carex (Carex macloviana D'Urv.), entre otras. La zona sureste del área de estudio está bajo concesión minera para explotación de turba, que consiste en 3 fragmentos que suman en total 134 ha. La extracción minera se desarrolla en franjas paralelas (trincheras) de aproximadamente 10-15 m de ancho, alternadas con franjas sin explotar. En la zona de explotación se extrae la turba hasta aproximadamente 1,0 $\mathrm{m}$ de profundidad. Estas franjas están conectadas a canales periféricos utilizados para drenaje artificial. Los fragmentos de la zona noroeste en tanto, están sin intervenir. Esta zona tiene 11 fragmentos que suman 474 ha.

\section{REGISTRO DE CAMPO EN TURBERAS}

En los fragmentos no intervenidos por explotación minera se realizaron 43 censos fitosociológicos, para caracterizar atributos ecológicos de la vegetación y el sustrato de turberas. La selección de los puntos de muestreo fue aleatoria a partir de imágenes satelitales de los 11 fragmentos de turberas no intervenidas. Durante los meses de febrero a abril de 2018 , se muestreó la vegetación mediante dos procedimientos para caracterizar la flora y vegetación en: a) una escala espacial de rodal (hectáreas) y b) una escala espacial de micrositio. El registro de la vegetación a escala espacial de rodal consistió de parcelas de vegetación de $25 \mathrm{~m}^{2}$, donde se registraron las especies de plantas vasculares y no vasculares, y se estimó de manera visual la cobertura de cada una de ellas mediante escalas de porcentajes aproximados. El registro de la vegetación a escala de micrositio, en tanto, se cuantificó mediante una parcela de vegetación de $1 \mathrm{~m}^{2}$, localizada en el centro de la parcela mayor. Se registró también el listado florístico con escalas de porcentaje estimadas visualmente (Mueller-Dombois \& Ellenberg 1974) y se caracterizó el microrrelieve de la turbera clasificándola en una escala ordinal como llano (1), irregular ( \pm con cojines menores a $10 \mathrm{~cm}$ ) (2), y muy irregular (con cojines mayores a $20 \mathrm{~cm}$ ) (3), y se midió el $\mathrm{pH}$ de la vegetación ( $\mathrm{pH}$ meter PCE-PH2OS). En el centro de esta parcela se extrajo un testigo de $1 \mathrm{~m}$ de profundidad con un barreno de $5 \mathrm{~cm}$ de diámetro (Russian D-corer, De Vleeschouwer et al. 2010), con el fin de caracterizar el sustrato desde el punto de vista de su descomposición usando la escala de von Post (Von Post 1924, Clymo 1983) y pH-metro de campo (modelo PCE-PH2OS). El sustrato de $1 \mathrm{~m}$ fue dividido en subsecciones que se diferenciaban por capas con diferentes grados de descomposición $\left(\mathrm{O}_{i}, \mathrm{O}_{\mathrm{e}}, \mathrm{O}_{\mathrm{a}}\right)$, cada uno de los cuales fue medido en su profundidad y $\mathrm{pH}$. La determinación de las plantas vasculares, no vasculares y líquenes, se realizó mediante el uso de claves taxonómicas y guías de campo (Moore 1983, Vidal 2006, Domínguez 2012, Larraín 2007), y las exsicatas fueron depositadas en el Herbario del Instituto de la Patagonia (HIP). La nomenclatura siguió a Rodríguez et al. (2018).

\section{CLASIFICACIÓN DE COBERTURAS DE VEGETACIÓN A ESCALA ESPACIAL}

Se utilizaron datos satelitales worldview-2 (adquiridos el 13 de mayo de 2016), con una resolución espacial de $2 \mathrm{~m}$ para las bandas multiespectrales, y de $0,5 \mathrm{~m}$ para la banda pancromática. Por otro lado, dichas imágenes poseen 4 bandas espectrales (azul, verde, rojo e infrarrojo cercano). La leyenda definida para la presente clasificación consideró la identificación de 3 clases de vegetación incluyendo turberas camefíticas + turberas graminoides (ambas fusionadas), turberas esfagnosas y turberas pulvinadas. Además, se clasificó una cuarta categoría definida como sectores con presencia de agua y/o sombra proyectada por árboles adyacentes a la comunidad turbosa. Se combinaron técnicas de procesamiento digital de imágenes, en conjunto con la información de terreno. Se aplicó una clasificación supervisada orientada al píxel, para lo cual fue necesario generar áreas de entrenamiento, aplicar un algoritmo de asignación y un método de validación de la clasificación obtenida. Las áreas de entrenamiento corresponden a áreas representativas de la clase que se desea extraer. Por lo tanto, una buena clasificación depende directamente de la calidad de los datos de entrenamiento, siendo aún más importantes en clasificaciones basadas en imágenes de alta resolución espacial (Chen \& Stow 2002). En el presente estudio se generaron a partir de interpretación visual en complemento con conocimiento experto del área de estudio. Se generaron aproximadamente 100 píxeles de entrenamiento para cada clase definida, distribuidos de manera heterogénea sobre 
el área de estudio. Existen varias técnicas de asignación automática de un píxel a una clase determinada de acuerdo con estadísticas de las áreas de entrenamiento. Una de las más utilizadas en teledetección de datos provenientes de sensores ópticos, es el método de máxima verosimilitud (Miao et al. 2011, Yonezawa 2007). Este clasificador asume que los datos siguen una distribución normal, de esta manera se puede describir una clase a partir de una función de probabilidad y así poder determinar la probabilidad de que un píxel pertenezca a una de las clases caracterizadas por las áreas de entrenamiento previamente definidas. Aplicando este método a una imagen es posible asignar todos los píxeles a las clases consideradas en la leyenda definida, obteniendo así nuestra imagen clasificada. La fase final del proceso de clasificación de los datos corresponde a la validación de la imagen obtenida luego de aplicar el algoritmo de asignación y tiene como objetivo comprobar la validez y exactitud del método y el producto generado. En general, la evaluación de la exactitud de la clasificación es medida a través del grado de correspondencia entre la etiqueta categórica predicha de los píxeles en la imagen clasificada, y datos observados o de referencia para cada clase (Foody 2005). Para la validación de la imagen clasificada se utilizaron los datos obtenidos en las campañas de campo. Se generaron matrices de confusión (valores predichos $\mathrm{v} / \mathrm{s}$ verdad de terreno) y se calculó la fiabilidad global como indicador de exactitud.

\section{ANÁLISIS ESTADÍSTICOS}

Los atributos estructurales del sustrato se caracterizaron y agruparon en 9 variables que incluyeron descomposición de la turba (escala von Post, Clymo 1983), promedio de descomposición de sustratos a $1 \mathrm{~m}$ de profundidad, $\mathrm{pH}$ de la capa de baja descomposición $\mathrm{O}_{\text {, }} \mathrm{pH}$ promedio de los sustratos a $1 \mathrm{~m}$ de profundidad, profundidad de la capa Oi, microtopografía rankeada $(1=\|$ lano; $2=$ irregular; $3=$ muy irregular), cobertura total de la vegetación, cobertura de agua y suelo desnudo. Estas variables fueron agrupadas de acuerdo a los tipos de vegetación para evaluar diferencias estadísticamente significativas entre los grupos, mediante la prueba Kruskal-Wallis y la prueba Dunns para comparaciones múltiples (Sokal \& Rohlf 1980). Se caracterizaron atributos composicionales de la vegetación de los diferentes tipos de turberas expresadas como riqueza promedio de plantas, riqueza promedio de plantas vasculares, riqueza promedio de plantas no vasculares y líquenes, índice de Shannon e índice de equitatividad. Estos indicadores fueron comparados entre tipos de turberas para evaluar diferencias estadísticamente marginales (i.e. $P<0,01)$ mediante la prueba Kruskal-Wallis y la prueba Dunns para comparaciones múltiples. Además, se generaron otros indicadores como los valores de importancia
(Cottam \& Curtis 1956) para las 7 especies más comunes. Se determinaron los espectros biológicos para cada tipo de turbera, promediando las abundancias absolutas de las diferentes formas de vida sobre la base del sistema Raunkiaer (Mueller-Dombois \& Ellenberg 1974), excluyendo al musgo Sphagnum cuya forma de vida no es clasificada en este sistema. Finalmente, se determinaron especies diagnósticas o con preferencia por uno o varios grupos de vegetación, mediante el análisis de especies indicadoras (De Cáceres et al. 2010), sobre los datos de abundancia transformados a logaritmo (log [abundancias + 1]). Para evaluar similitud florística entre grupos (e.g. diversidad beta), se exploró la estructura multivariada conjunta de los censos en el espacio de las especies, mediante el modelo de ordenación "Escalamiento Multidimensional No Métrico" (NMDS) (Clarke \& Aisworth 1993). Las variables (especies) fueron transformadas a logaritmo y se usó BrayCurtis como distancia ecológica. La ordenación de los censos se ajustó a 12 variables ambientales incluyendo cobertura de agua, cobertura total, microtopografía, $\mathrm{pH}$ promedio de los sustratos a $1 \mathrm{~m}$ de profundidad, $\mathrm{pH}$ de la capa Oi, profundidad de la capa Oi, riqueza de plantas no vasculares y líquenes, riqueza de plantas vasculares, riqueza de plantas, promedio de descomposición de sustratos a $1 \mathrm{~m}$ de profundidad (von Post), suelo desnudo, descomposición de la capa Oi (von Post). Los puntajes obtenidos para las variables ambientales fueron correlacionados con los puntajes de los censos de vegetación mediante correlación de Pearson (Sokal \& Rohlf 1980), con la finalidad de interpretar los principales gradientes ambientales que determinan la distribución de la vegetación. Se comparó la disimilitud intragrupo y entre grupos mediante la prueba no paramétrica "Análisis de Similitud" (ANOSIM, Clarke 1993) usando distancia Bray-Curtis sobre los datos de abundancia transformados a logaritmo. Las comparaciones múltiples se realizaron mediante la prueba de corrección óptima de Bonferroni (Clarke 1993).

\section{RESULTADOS}

\section{Atributos espaciales DE LAS tURBERAS}

La clasificación espacial resultante de los diferentes tipos de turberas obtuvo una fiabilidad global de un $67 \%$. Cabe destacar que la mayor confusión en la clasificación se produjo entre las clases turberas camefíticas + turberas gaminoides y turberas esfagnosas. La clase con mayor superficie resultó ser las turberas camefíticas + turberas graminoides, cuya separación según píxeles no fue posible de determinar. Estos dos tipos de vegetación suman 350,1 ha con 875.142 píxeles. La clase turberas esfagnosas, en tanto, resultó con una superficie total de 78,1 ha con 195.208 píxeles. La clase 
turberas pulvinadas obtuvo la menor superficie, con sólo 5,0 ha y 12.575 píxeles (Tabla 1). Finalmente, la categoría correspondiente a agua y sombra, obtuvo 13,2 ha y 33.031 píxeles. La imagen resultante muestra la distribución de los diferentes tipos de turberas en el sector "La Grazzia" (Fig 1).

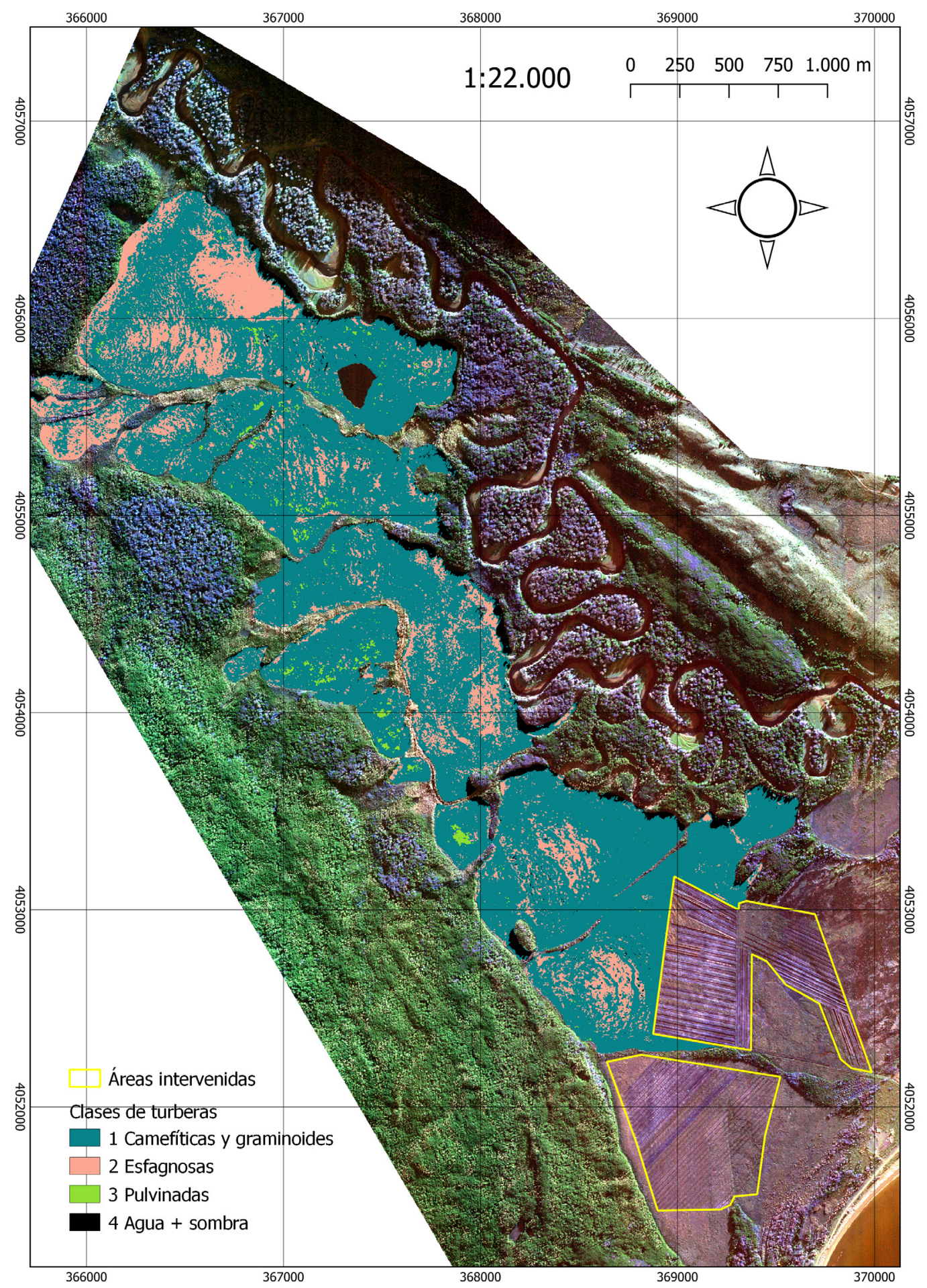

Figura 1. Mapa de superficies clasificadas según conocimiento experto y sistema de clasificación remota, para los diferentes tipos de vegetación de turberas ombrogénicas del sector "La Grazzia" (Península de Brunswick, Magallanes). / Map of classified areas according to expert knowledge and remote classification system, for the different types of vegetation of ombrogenic peat bogs in the "La Grazzia" sector (Brunswick Peninsula, Magallanes). 
TABLA 1. Superficies clasificadas según conocimiento experto y sistema de clasificación remota, para los diferentes tipos de turberas ombrogénicas del sector "La Grazzia" (Península de Brunswick, Magallanes). / Surfaces classified according to expert knowledge and remote classification system, for the different types of vegetation of ombrogenic peat bogs in the "La Grazzia" sector (Brunswick Península, Magallanes).

\begin{tabular}{lcccc}
\hline Clase & Código & $\begin{array}{c}\text { Píxeles } \\
\text { (n) }\end{array}$ & $\begin{array}{c}\text { Superficie } \\
\text { (ha) }\end{array}$ & $\begin{array}{c}\text { Proporción } \\
\text { (\%) }\end{array}$ \\
\hline Turbera graminoide + turbera camefítica & gram + came & 875.142 & 350,1 & 78,4 \\
Turbera esfagnosa & esfa & 195.208 & 78,1 & 17,5 \\
Turbera pulvinada & pulv & 12.575 & 5,0 & 1,1 \\
Agua + sombra & agua + somb & 33.031 & 13,2 & 3,0 \\
\hline Total & & 1.115 .956 & 446,4 & 100,0 \\
\hline
\end{tabular}

\section{Atributos estructurales del sustrato}

La descomposición de la capa Oi y de las capas promedio Oi, Oe y $\mathrm{Oa}$ a $1 \mathrm{~m}$ de profundidad, de acuerdo a la escala de von Post, fue significativamente mayor en las turberas pulvinadas, que en promedio $( \pm E S)$ tuvieron $7,25( \pm 0,75)$ y $8,0( \pm 0,56)$, respectivamente. Los menores valores de descomposición se hallaron en las turberas esfagnosas que, tanto para la capa Oi como para las capas $\mathrm{Oi}$, Oe y Oa, tuvieron valores de 3,4 $( \pm 0,3)$ y $4,4( \pm 0,2)$, respectivamente (Figs. 2 a, 2 b). Por otra parte, el $\mathrm{pH}$ tanto en la capa $\mathrm{Oi}$, como el $\mathrm{pH}$ promedio de las capas a $1 \mathrm{~m}$ de profundidad, mostró valores relativamente bajos para todas las turberas, fluctuando desde 3,12 a 4,22 y 3,33 a 4,83, respectivamente. No se observaron diferencias estadísticamente significativas entre el $\mathrm{pH}$ para los diferentes grupos de turberas (Figs. 2c, 2d). La profundidad de la capa Oi fluctuó entre 10 y $100 \mathrm{~cm}$, y al igual que en el pH no se registraron diferencias estadísticamente significativas entre los tipos de vegetación (Fig. 2e). Con respecto a la microtopografía, los mayores valores se registraron para las turberas graminoides y las camefíticas, con promedios de $2,6( \pm 0,2)$ y $2,5( \pm 0,7)$, respectivamente. Los menores valores de microtopografía se registraron en las turberas pulvinadas, con un promedio de $1,2( \pm 0,2)$. Se registraron diferencias estadísticamente significativas entre los grupos (Fig. 2f). La cobertura total de la vegetación fue mayor en las turberas graminoides, con valores promedios de 98,7 $( \pm 1,2)$. Las turberas esfagnosas, por otra parte, registraron los valores promedios más bajos de 84,7 ( $\pm 6,0)$. Se evidenciaron diferencias estadísticas significativas entre los grupos de vegetación (Fig. $2 \mathrm{~g}$ ). La variable cobertura de agua mostró tener los mayores valores en las turberas pulvinadas, con una cobertura promedio de $12,5( \pm 7,2)$. Las turberas graminoides, camefíticas y esfagnosas no presentaron cobertura de agua. Se registraron diferencias estadísticamente significativas entre las turberas pulvinadas y el resto de los grupos de vegetación (Fig. 2h). Finalmente, el suelo desnudo mostró las mayores coberturas en las turberas esfagnosas, con un promedio de $9,0( \pm 2,2)$. Las turberas graminoides en tanto, mostraron los valores más bajos para esta variable, con valores promedio de $1,2( \pm 1,2)$. Las comparaciones múltiples mostraron diferencias estadísticamente significativas entre los grupos (Fig. 2i).

Patrones de estructura multivariada conjunta $\mathrm{Y}$ Disimilitud ENTRE TIPOS DE TURBERAS

La ordenación multivariada en el espacio de las especies mostró que los ejes principales separaron adecuadamente los grupos de turberas determinados con conocimiento experto. El Stress del NMDS fue bajo (Stress=0,1511), indicando una estructura estable (Fig. 3). Asimismo, la varianza total explicada por los 2 ejes principales fue de $r^{2}=0,9049$. Seis variables ambientales, relacionadas con la vegetación y sustrato se correlacionaron significativamente con el eje principal. Las variables positivamente correlacionadas fueron cobertura de agua ( $r=0,43 ; P<0,0001$ ), riqueza de plantas no vasculares y líquenes ( $r=0,42 ; P<0,0001)$, riqueza total de plantas $(r=0,39$; $P<0,001)$ y riqueza de plantas vasculares $(r=0,27 ; P<0,01)$, mientras que las variables negativamente correlacionadas con el eje fueron la microtopografía $(r=-0,44 ; P<0,0001)$ y cobertura total de la vegetación $(r=-0,35 ; P<0,01)$ (Tabla 2). Por otra parte, cinco variables se correlacionaron con el eje secundario. Las variables positivamente correlacionadas fueron $\mathrm{pH}$ del horizonte $\mathrm{o}(r=0,36 ; P<0,001), \mathrm{pH}$ promedio de los sustratos a $1 \mathrm{~m}$ de profundidad $(r=0,31 ; P<0,01)$ y riqueza de plantas vasculares $(r=0,25 ; P<0,05)$. Por otra parte, las variables negativamente correlacionadas con el eje secundario fueron descomposición en la capa Oi $(r=-0,49$; $P<0,0001)$ y promedio de descomposición de capas $\mathrm{Oi}$, Oe y Oa a $1 \mathrm{~m}$ de profundidad ( $r=-0,42 ; P<0,0001)$ (Tabla 2). 
Finalmente, el ANOSIM mostró que la disimilitud entre grupos (media rankeada $=2,116$ ) fue mayor que las disimilitudes dentro de los grupos (media rankeada=1,141) $(R=0,5335$; $P<0,0001)$. Las comparaciones múltiples mostraron que la mayor disimilitud se registró entre las turberas graminoides y las turberas pulvinadas ( $R=0,9367 ; P=0,0012)$, mientras que la menor disimilitud se registró entre las turberas esfagnosas y las camefíticas $(R=0,3064 ; P=0,0006)$ (Tabla 3 ). a) Descomposición capa Oi

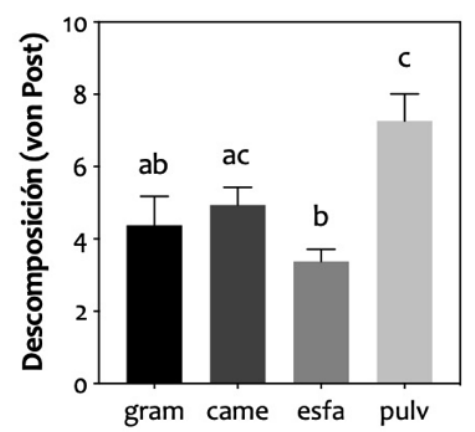

d) $\mathrm{pH}$ capas $1 \mathrm{~m}$

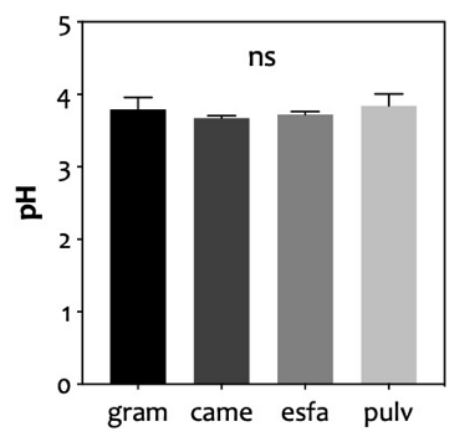

g) Cobertura total

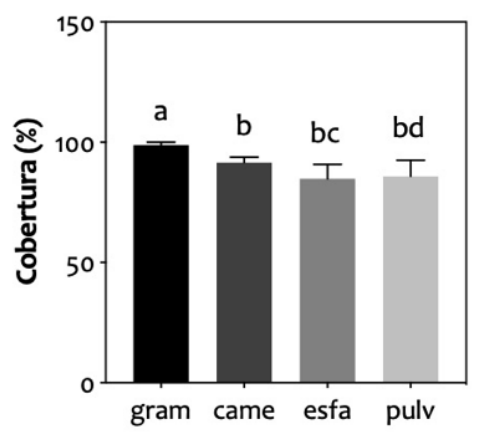

b) Descomposición $1 \mathrm{~m}$

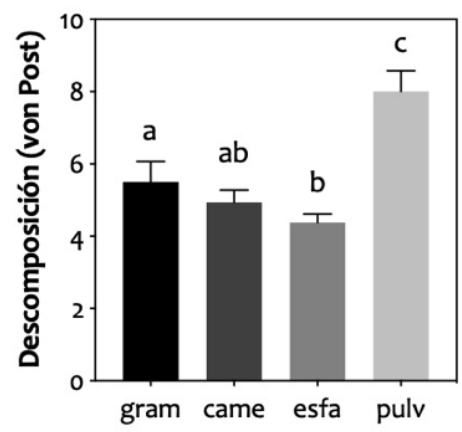

e) Profundidad capa Oi

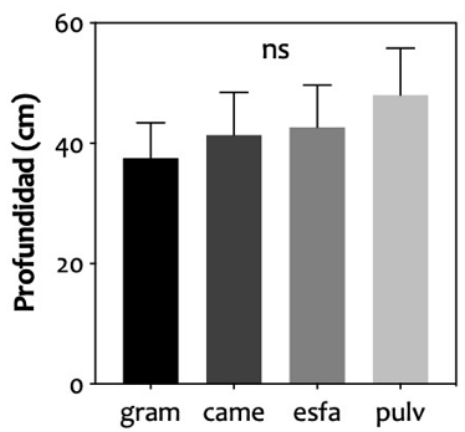

h) Cobertura agua

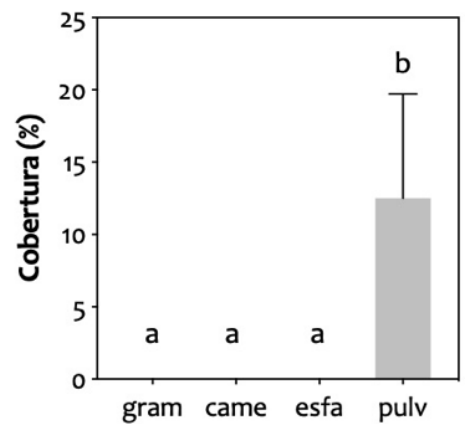

c) $\mathrm{pH}$ capa Oi

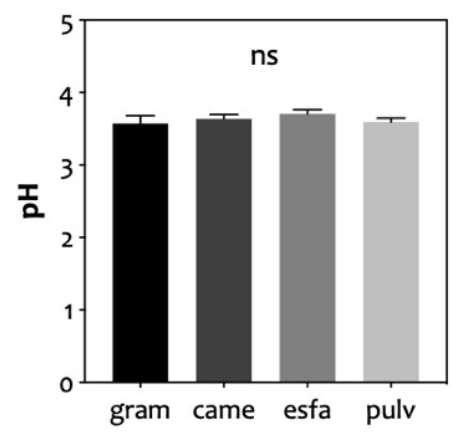

f) Microtopografía

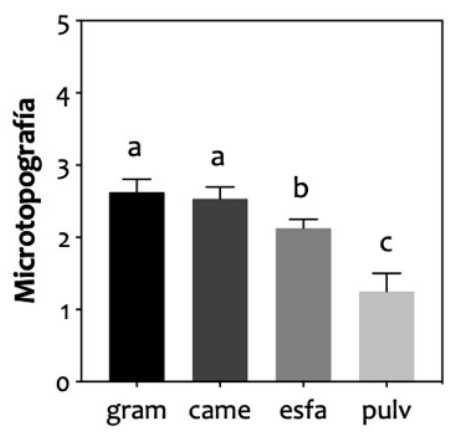

i) Suelo desnudo

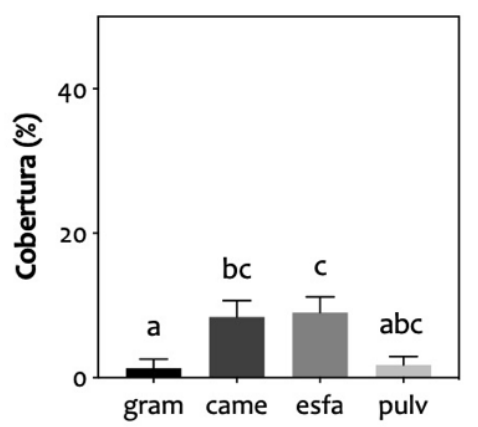

Figura 2. Resumen de variables ambientales para los diferentes tipos de vegetación de turberas en el sector "La Grazzia" (Península de Brunswick, Magallanes). Se indican diferencias estadísticamente significativas para las variables entre tipos de vegetación (prueba K-W, $P<0,05)$. / Summary of environmental variables for different vegetation of peat bogs in the "La Grazzia" sector (Brunswick Peninsula, Magallanes). Statistically significant differences are indicated for the variables between vegetation types $(K-W$ test, $P<0.05)$. 


\section{NMDS vegetación turberas}

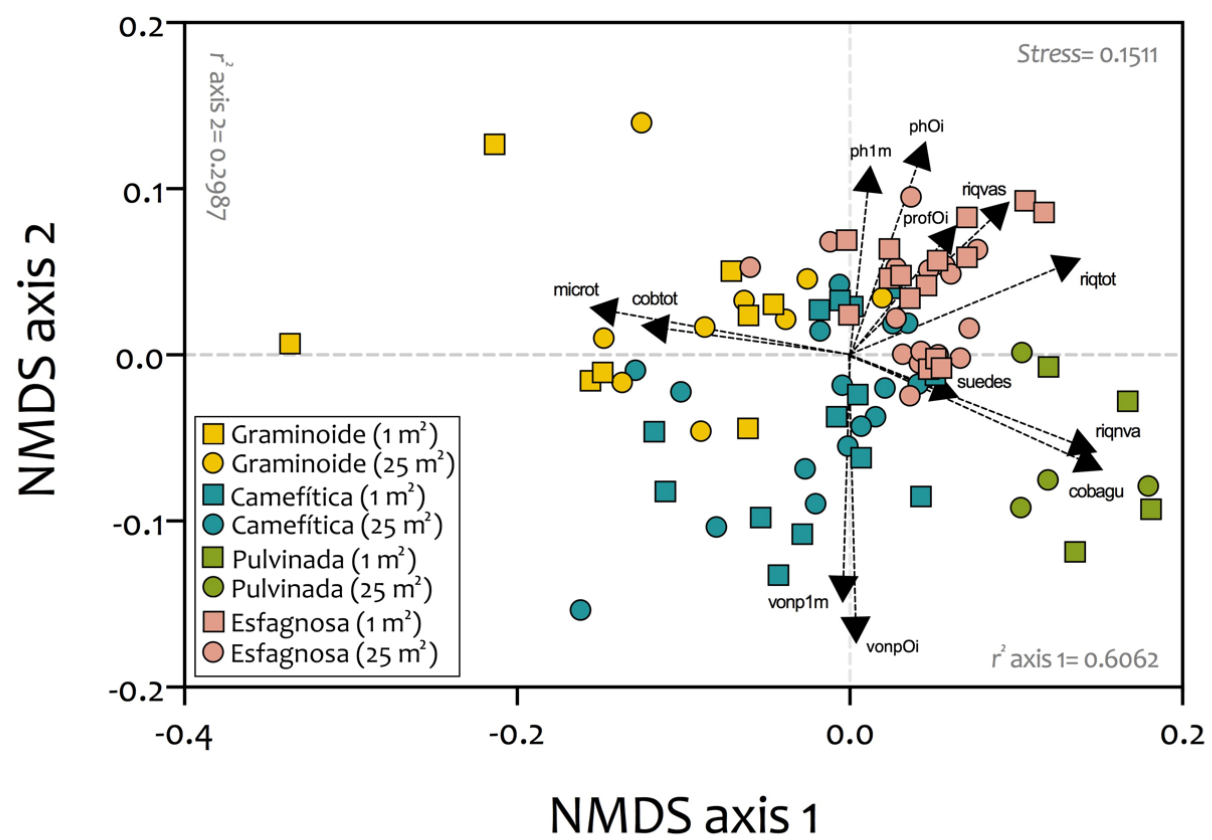

FigURA 3. Ordenación multivariada (NMDS) para la similitud florística entre censos de turberas ombrogénicas en el sector "La Grazzia" (Península de Brunswick, Magallanes). Las abreviaturas de las variables ambientales son indicadas (cobagu= cobertura de agua; cobtot= cobertura total de la vegetación; microt= microtopografía rankeada; $\mathrm{ph} 1 \mathrm{~m}=\mathrm{pH}$ promedio de las capas a $1 \mathrm{~m}$ de profundidad; phOi= pH de la capa Oi; profOi= profundidad de la capa Oi; riqnva= riqueza plantas no vasculares y líquenes; riqtot= riqueza total de plantas; riqvas= riqueza de vasculares; suedes= suelo desnudo; vonp $1 \mathrm{~m}=$ promedio de descomposición de sustratos a $1 \mathrm{~m}$ de profundidad; vonpOi= descomposición de la capa Oi). / Multivariate ordination (NMDS) for floristic similarity between ombrogenic peatland plots in the "La Grazzia" sector (Brunswick Peninsula, Magallanes). The abbreviations of the environmental variables are indicated (cobagu $=$ water coverage, cobtot $=$ total coverage of the vegetation, microt $=$ microtopography, phO1 $=$ average $\mathrm{pH}$ of the substrates at 1 $\mathrm{m}$ depth, $\mathrm{phOi}=\mathrm{pH}$ of the horizon Oi; profOi $=$ depth Oi; riqnva = non-vascular plant richness; riqtot $=$ total plant richness; riqvas = vascular richness; soils = bare soil cover; vonp $1 \mathrm{~m}=$ average decomposition of substrata at $1 \mathrm{~m}$ depth; vonpOi = decomposition at Oi).

TABLA 2. Correlaciones de variables ecológicas (variables de la vegetación y del sustrato) con los ejes principales obtenidos en la ordenación NMDS (correlación Pearson, $r ;^{*}=P<0,05 ;{ }^{* *}=P<0,01 ;{ }^{* * *}=P<0,001 ;{ }^{* * * *}=P<0,0001$ ). / Correlations of ecological variables (vegetation and substrate variables) with the principal axes obtained in the NMDS ordination (Pearson correlation, $r ;{ }^{*}=P<0.05 ;{ }^{* *}=P$ $\left.<0.01 ;^{* * *}=P<0.001 ;{ }^{* * * *}=P<0.0001\right)$.

\begin{tabular}{lcl}
\hline Variables ecológicas & Axis 1 & Axis 2 \\
\hline Variables de vegetación & & \\
Cobertura total vegetación & $-0,3461^{* *}$ & 0,05267 \\
Riqueza no vasculares & $0,4155^{\cdots * *}$ & 0,166 \\
Riqueza total & $0,3878^{* * *}$ & 0,1633 \\
Riqueza vasculares & $0,2663^{* *}$ & $0,2547^{*}$ \\
Variables de sustrato & & \\
Cobertura de agua & $0,4302^{* * * *}$ & $-0,1946$ \\
Microtopografía & $-0,4388^{* * * *}$ & 0,08028 \\
pH 1 metro & 0,0332 & $0,3138^{* *}$ \\
pH capa Oi & 0,1267 & $0,3577^{* * *}$ \\
pH vegetación & $-0,06364$ & 0,1147 \\
Profundidad capa Oi & 0,1732 & 0,2105 \\
Suelo desnudo & 0,1959 & 0,07514 \\
von Post 1 m & 0,00549 & $-0,418^{* * * *}$ \\
von Post capa Oi & 0,008954 & $-0,4907^{* * *}$ \\
\hline
\end{tabular}


TABLA 3. Resultados del Análisis de Similitud (ANOSIM) sobre la comparación múltiple entre diferentes tipos de turberas en el sector "La Grazzia" (Península de Brunswick, Magallanes). Se indican los valores de disimilitud $(R)$ entre tipos de vegetación en la sección superior derecha de la matriz, y los valores $P$ (corrección Bonferroni). / Results of the Analysis of Similarity (ANOSIM) on the multiple comparison between different types of vegetation peat bogs in the sector of "La Grazzia" (Brunswick Peninsula, Magallanes). The dissimilarity values $(R)$ between types of vegetation in the upper right section of the matrix, and the $P$ values (Bonferroni correction) are indicated.

\begin{tabular}{lcccc}
\hline & gram & came & esfa & pulv \\
\hline gram & & 0,5137 & 0,8303 & 0,9367 \\
came & 0,0006 & & 0,3064 & 0,8066 \\
esfa & 0,0006 & 0,0006 & & 0,925 \\
pulv & 0,0012 & 0,0006 & 0,0006 & \\
\hline
\end{tabular}

\section{Atributos de COMPOSICIÓN FLORÍSTICA DE LAS TURBERAS}

La riqueza total de especies de plantas fue mayor en las turberas graminoides, con un total de 21 especies. En orden decreciente, la riqueza total registrada fue de 18 especies en las turberas camefíticas, 17 en las turberas esfagnosas y 14 en las turberas pulvinadas (Tabla 4, Anexo 1). Sin embargo, los valores más altos para la riqueza promedio total de plantas ( \pm ES) fue registrada en las turberas pulvinadas $(7,5 \pm 2,0)$. Le siguieron las turberas esfagnosas (5,9 $\pm 1,3)$, las turberas graminoides $(5,1 \pm 2,1)$ y finalmente las turberas camefíticas $(4,7 \pm 1,2)$ (Tabla 4). Así mismo, se observaron diferencias estadísticamente significativas en las comparaciones múltiples, donde la riqueza de las turberas pulvinadas fue comparativamente mayor. Por otra parte, la riqueza promedio de plantas vasculares fue mayor en las turberas pulvinadas $(6,0 \pm 0,8)$, seguidas por las turberas esfagnosas $(4,6 \pm 1,3)$, las turberas camefíticas $(3,5 \pm 1,0)$ y las turberas graminoides (1,4 $\pm 1,7)$, con diferencias estadísticamente significativas según las comparaciones múltiples. La riqueza promedio de plantas no vasculares y líquenes fue en general baja, no encontrándose diferencias significativas en las comparaciones entre grupos. El índice de Shannon registró valores relativamente bajos de biodiversidad, sin encontrarse diferencias entre grupos para la métrica. El índice de Equitatividad, en tanto, fue comparativamente mayor en las turberas graminoides $\left(J^{\prime}=0,68 \pm 0,05\right)$, seguido por las turberas camefíticas $\left(J^{\prime}=0,58 \pm 0,04\right)$, las turberas esfagnosas $\left(J^{\prime}=0,51 \pm 0,03\right)$ y las turberas pulvinadas $\left(J^{\prime}=0,43 \pm 0,05\right)$ (Tabla 4). Las ocho especies más relevantes, de acuerdo al valor de importancia fueron Cladonia spp., Empetrum rubrum, Gaultheria antarctica, Marsippospermum grandiflorum, Nothofagus betuloides, Sphagnum magellanicum y Tetroncium magellanicum. Los valores de importancia son descritos en la Tabla 4. Las especies diagnósticas para uno u otro tipo de turberas en tanto, fueron Caltha appendiculata, Donatia fascicularis, Drosera uniflora, Marsippospermum grandiflorum,
Myrteola nummularia y Tetroncium magellanicum. Los valores ISA y preferencias por tipo de vegetación son indicados en la Tabla 4.

\section{ESPECTROS BIOLÓGICOS DE LAS TURBERAS}

Con respecto a las formas de vida y los espectros biológicos, los fanerófitos resultaron ser dominantes en la turbera graminoide con un promedio ( \pm ES) de cobertura de 12,0 ( \pm 9,12) (Fig. 4). Por otra parte, los caméfitos tuvieron coberturas dominantes en las turberas camefíticas y en las turberas pulvinadas, con promedios de 56,0 ( $\pm 5,9)$ y 58,0 ( $\pm 2,7)$, respectivamente. Los hemicriptófitos dominaron especialmente en las turberas esfagnosas, con promedio de cobertura de 13,4 ( $\pm 3,0)$. Por otra parte, los criptófitos dominaron las turberas graminoides con promedio de cobertura de 41,9 ( $\pm 4,3$ ).

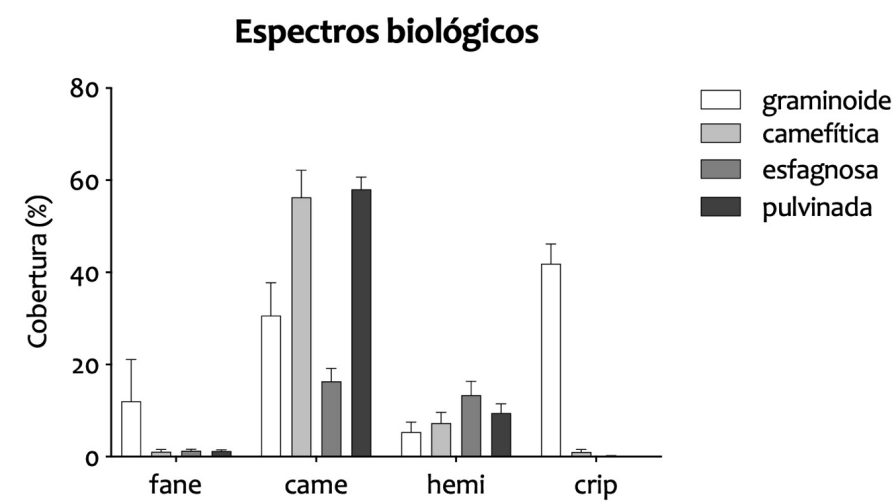

Figura 4. Espectros biológicos de los diferentes tipos de turberas en el sector "La Grazzia" (Península de Brunswick, Magallanes). Formas de vida: fane= fanerófitos; came= caméfitos; hemi= hemicriptófitos; crip= criptófitos. / Biological spectra of the different vegetation types of peat bogs in the "La Grazzia" sector (Brunswick Península, Magallanes). Life forms: fane = phanerophyte; came $=$ chamaephyte; hemi $=$ hemicryptophyte; crip = cryptophyte. 
TABla 4. Indicadores de composición de la vegetación para los diferentes tipos de turberas en el sector "La Grazzia" (Península de Brunswick, Magallanes). Se indica además los valores de importancia para las 8 especies más comunes y las 6 especies diagnósticas de acuerdo al valor ISA. Se indican diferencias estadísticamente marginales para las variables riqueza promedio total de plantas, riqueza promedio plantas vasculares y riqueza promedio plantas no vasculares y líquenes, índice de Shannon y equitatividad (prueba $K$-W, $P<$ $0,01)$. Para las especies diagnósticas se muestra el valor ISA y la significancia estadística $\left(P<0,05={ }^{*} ; P<0,01={ }^{* *} ; P<0,001={ }^{* * *}\right)$. / Indicators of vegetation composition for the different types of peat bogs in the "La Grazzia" sector (Brunswick Peninsula, Magallanes). It also indicates the values of importance for the 8 most common species and the 6 diagnostic species according to the ISA value. Statistically marginal differences are indicated for the variables total average plant richness, average vascular plant richness and average nonvascular plant richness, Shannon index and equitativity ( $\mathrm{K}-\mathrm{W}$ test, $\mathrm{P}<0.01)$. For the diagnostic species, the ISA value and statistical significance are shown $\left(P<0.05=^{*}, P<0.01=^{* *}, P<0.001=^{* * *}\right)$.

\begin{tabular}{|c|c|c|c|c|}
\hline Indicadores composición & $\begin{array}{c}\text { Turbera } \\
\text { Graminoidea }\end{array}$ & $\begin{array}{c}\text { Turbera } \\
\text { camefítica }\end{array}$ & $\begin{array}{c}\text { Turbera } \\
\text { esfagnosa }\end{array}$ & $\begin{array}{c}\text { Turbera } \\
\text { pulviniforme }\end{array}$ \\
\hline Número de censos & 8 & 15 & 16 & 4 \\
\hline Número total de especies & 21 & 18 & 17 & 14 \\
\hline Número total especies vasculares & 18 & 13 & 12 & 12 \\
\hline Número total especies no vasculares y líquenes & 3 & 5 & 5 & 2 \\
\hline Riqueza promedio ( \pm ES) total de plantas & $5,1(2,1)^{\mathrm{ab}}$ & $4,7(1,2)^{a}$ & $5,9(1,3)^{b}$ & $7,5(2,0)^{c}$ \\
\hline Riqueza promedio ( \pm ES) plantas vasculares & $1,4(1,7)^{\mathrm{ab}}$ & $3,5(1,0)^{a}$ & $4,6(1,3)^{b}$ & $6,0(0,8)^{c}$ \\
\hline Riqueza promedio ( \pm Es) plantas no vasculares & $1,0(0,8)^{\mathrm{ns}}$ & $1,3(0,9)^{\mathrm{ns}}$ & $1,4(0,5)^{\mathrm{ns}}$ & $1,5(0,6)^{\mathrm{ns}}$ \\
\hline Indice de Shannon & $1.06(0,12)^{\mathrm{ns}}$ & $0,76(0,12)^{\mathrm{ns}}$ & $0,99(0,06)^{\text {ns }}$ & $1,03(0,21)^{\text {ns }}$ \\
\hline Indice de equitatividad & $0,68(0,05)^{a}$ & $0,58(0,04)^{a b}$ & $0,51(0,03)^{b}$ & $0,43(0,05)^{b}$ \\
\hline Valor de importancia Cladonia spp. & 8,2 & 22,1 & 12,9 & 17,6 \\
\hline Valor de importancia Donatia fascicularis & 0,0 & 0,0 & 0,0 & 70,4 \\
\hline Valor de importancia Empetrum rubrum & 45,3 & 81,5 & 35,1 & 20,8 \\
\hline Valor de importancia Gaultheria antarctica & 15,1 & 21,7 & 18,8 & 10,9 \\
\hline Valor de importancia Marsippospermum grandiflorum & 62,1 & 5,3 & 1,2 & 0,0 \\
\hline Valor de importancia Nothofagus betuloides & 13,1 & 4,8 & 6,8 & 11,2 \\
\hline Valor de importancia Sphagnum magellanicum & 20,4 & 29,9 & 75,6 & 22,0 \\
\hline Valor de importancia Tetroncium magellanicum & 0,0 & 6,7 & 20,3 & 15,3 \\
\hline Valor de importancia sumado 8 especies principales & 164,2 & 172,0 & 170,7 & 168,2 \\
\hline Valor indicador (ISA) Caltha appendiculata & - & - & - & $0,628^{*}$ \\
\hline Valor indicador (ISA) Donatia fascicularis & - & - & - & $1,0^{* * *}$ \\
\hline Valor indicador (ISA) Drosera uniflora & - & - & $0,673^{*}$ & $0,673^{*}$ \\
\hline Valor indicador (ISA) Marsippospermum grandiflorum & $0,987^{* * *}$ & - & - & - \\
\hline Valor indicador (ISA) Myrteola nummularia & - & - & - & $0,640^{*}$ \\
\hline Valor indicador (ISA) Tetroncium magellanicum & - & - & $0,857^{* * *}$ & $0,857^{* * *}$ \\
\hline
\end{tabular}

\section{DISCUSIÓN}

Clasificación de las turberas ombrogénicas Y GRADIENTES ECOLÓGICOS

La clasificación vegetacional propuesta en este trabajo es consistente con previas clasificaciones fitogeográficas y fitosociológicas disponibles en la literatura. Por ejemplo, una reciente actualización sintaxonómica de la vegetación de turberas de la ecorregión Valdiviano-Magallánica (sensu Cabrera \& Willink 1973), propuso 13 asociaciones fitosociológicas dentro de la clase Myrteolo-Sphagnetea magellanici (Amigo et al. 2017). Estas asociaciones se incluyen dentro de grupos fisionómicos que describen turberas pulvinadas, turberas esfagnosas, turberas cípero-graminoides y turberas de tundra de montaña (e.g. Bonarelli 1917, Oberdorfer 1960, Pisano 1977, Dollenz 1980, Kleinebecker et al. 2007). En particular, nuestra tipificación coincide 
parcialmente con Amigo et al. (2017), quienes propusieron el sintaxón Nanoneo muscosae-Sphagnetum magellanici para las aquí denominadas turberas esfagnosas. Similarmente, la turbera pulvinada concuerda con la asociación Drosero uniflorae-Donatietum fascicularis, mientras que las turberas camefíticas son similares en composición y fisionomía al Gaultherio-Sphagnetum magellanici (Amigo et al. 2017). Las turberas graminoides, en tanto, con dominancia de Marsippospermum grandiflorum, requieren de ulteriores estudios para poderlas catalogar dentro de un contexto fitosociológico (Amigo et al. 2017). Coincidentemente, la reciente clasificación reportada por Mathijssen et al. (2019), basada en atributos fisionómicos de la vegetación, reportó las mismas unidades de vegetación descritas en este estudio.

Estudios recientemente publicados dan cuenta de la importancia de la composición florística en ambientes heterogéneos de turberas, debido a que proporcionan información relevante en relación con procesos ecológicos (secuestro de carbono, emisiones de metano, etc.) (PérezHaase et al. 2019, Mathijssen et al. 2019). Consecuentemente el uso de técnicas de percepción remota se presenta como una herramienta adecuada para el mapeo de distintos ambientes en turberas. El uso de imágenes satelitales de alta resolución espacial, como la utilizada en este estudio (Worldview-2), son sensibles a las variaciones florísticas a escalas cartográficas inferiores a 1:5000 (sensu Tobler 1987). Cabe mencionar que la percepción remota no sólo se limita al uso de imágenes satelitales, sino que también incluye datos obtenidos por aeronaves y más recientemente unidades aéreas no tripuladas; estas últimas permiten, inclusive, identificar plantas individuales, extendiendo las posibilidades en la identificación de tipos de vegetación en ambientes de turberas (Clutterbuck et al. 2018). Por otro lado, los datos Worldview-2 de 4 bandas multiespectrales (azul, verde, rojo e infrarrojo cercano) usados en este estudio, poseen principalmente una sensibilidad a los procesos de absorción de energía en el espectro azul $(478 \mathrm{~nm})$ y rojo $(649 \mathrm{~nm})$. Esta sensibilidad está asociada a procesos fotosintéticos de las plantas y a la reflectividad de la energía en el espectro del infrarrojo cercano $(831 \mathrm{~nm})$, asociada esencialmente a la estructura celular interna de las hojas (Chuvieco 2008). En este sentido, el uso de bandas espectrales adicionales o sensores hiperespectrales pueden aumentar la capacidad para discriminar entre diferentes ambientes en turberas (Harris et al. 2015). Finalmente, la percepción remota es una herramienta fundamental a la hora de entender a una escala de paisaje estos ecosistemas y generar estimaciones más exactas de parámetros tales como flujos de carbono, temperatura y contenido de agua (Lees et al. 2018).

La ordenación multivariada permite inferir dos gradientes ecológicos principales. El primer gradiente denotado por el eje $X$ puede ser interpretado como un gradiente de terrestrialización a paludificación. Las turberas graminoides y camefíticas, localizadas principalmente en el lado negativo del eje $\mathrm{X}$ son turberas con mayor cobertura total de la vegetación y con microtopografía muy irregular, mientras que las turberas esfagnosas y pulvinadas localizadas en la sección positiva del eje $\mathrm{X}$, pueden inferirse como las zonas con mayor paludificación. Este gradiente ha sido connotado como uno de los más importantes en la formación y desarrollo de diferentes tipos de vegetación en turberas ombrogénicas. Para el caso de las turberas de "La Grazzia" podría también estar correlacionado con un gradiente de margen-centro de la turbera, con diferentes niveles de profundidad de la napa freática, pendiente, aireación y elevación, que determinan diferentes tipos de vegetación, como ha sido demostrado para turberas europeas (Wheeler \& Proctor 2000, Bragazza et al. 2005). El segundo gradiente, localizado a través del eje $Y$ de la ordenación, puede ser interpretado como un gradiente trófico. Aquí, las turberas pulvinadas y camefíticas están localizadas en la sección negativa del eje, y correlacionadas con las variables de descomposición del sustrato, cuyos valores de descomposición son mayores. Por otra parte, y en la sección positiva del eje $\mathrm{Y}$, las turberas esfagnosas $\mathrm{y}$ las graminoides tienen dominancia, con mayores valores de $\mathrm{pH}$ y menores valores de descomposición. Este resultado es coincidente con el estudio de Kleinebecker et al. (2007), quienes registraron un incremento en la descomposición para turberas pulvinadas de la zona archipielágica de Magallanes. Algunos estudios sugieren una positiva relación entre descomposición y disponibilidad de nutrientes (Clarkson et al. 2004, Bubier et al. 2007). Al mismo tiempo, numerosos factores se combinan para que la vegetación de turberas presente mayores o menores valores de descomposición del sustrato. Por ejemplo, las turberas dominadas por Sphagnum tienden a generar menor hojarasca y lenta descomposición, debido principalmente al carácter poroso del musgo, y sus altos contenidos de lignina y polifenoles que provoca una alta resistencia a la descomposición por microbios (Verhoeven \& Liefveld 1997) y a la humificación (Bonarelli 1917). Por el contrario, la vegetación leñosa de las turberas camefíticas y de las turberas pulvinadas, podría generar mayores niveles de hojarasca y consecuentemente incrementar la descomposición de la misma, aumentando la humificación. Por otra parte, aunque el eje $\mathrm{Y}$ está positivamente correlacionado con el $\mathrm{pH}$, no se evidenciaron diferencias estadísticamente significativas entre los diferentes tipos de turberas. Este resultado es similar al reportado por Clarkson et al. (2004), quienes estudiando turberas ombrotróficas dominadas por Sporadanthus y Empodisma (Restionaceae) en Nueva Zelanda, 
no encontraron diferencias para el $\mathrm{pH}$ de los diferentes tipos de vegetación clasificados en la sucesión de turberas. Al igual que en el estudio mencionado, el rango de $\mathrm{pH}$ reportado aquí es poco amplio (fuertemente ácido a moderadamente ácido, sensu Succow \& Joosten 2001), a diferencia de otros estudios de turberas del hemisferio norte donde se compararon turberas minerotróficas con turberas ombrotróficas (e.g. Vitt \& Chee 1990, Wheeler \& Proctor 2000). Así, los cambios en la vegetación de turberas ombrogénicas de "La Grazzia", no pueden ser atribuibles a cambios en el pH, y delatan en conjunto su carácter ombrotrófico.

\section{DISIMILITUD FLORÍSTICA ENTRE TIPOS DE TURBERAS}

Numerosos estudios locales y regionales han descrito los diferentes tipos de coberturas vegetaciones en turberas de Magallanes, con especial énfasis en clasificaciones fitogeográficas, y más recientemente fitosociológicas (e.g. Pisano 1977, Dollenz 1982, Henríquez 2004, Amigo et al. 2017). Sin embargo, estos estudios carecen en su mayoría de una aproximación numérica que permita inferir relaciones florísticas entre tales coberturas. Una aproximación de este tipo podría ser de ayuda para proponer patrones de sucesión de especies en gradientes ecológicos de importancia como niveles de la napa freática, suministro de nutrientes, gradientes microtopográficos, y gradientes de acidez-alcalinidad. Dollenz (1982), por ejemplo, da cuenta de que turberas musgosas de Sphagnum magellanicum y Sphagnum fimbriatum de la reserva nacional Magallanes, al ser desecadas de manera natural o artificial, podrían dar origen a otras comunidades, como aquellas dominadas por el musgo Polytrichum alpestre y ciperaceas como Marsippospermum grandiflorum. En nuestro estudio, la mayor disimilitud florística ocurrió entre las turberas pulvinadas y graminoides, mientras que las menores disimilitudes ocurrieron entre las turberas esfagnosas y camefíticas. La disimilitud registrada en este estudio nos permite proponer una cenoclina sucesional asociada a procesos de terrestrialización a paludificación que iría en sentido turberas graminoides, camefíticas, esfagnosas y pulvinadas, con especies típicamente asociadas al gradiente. Sin embargo, esta propuesta especulativa debe ser confirmada mediante futuros estudios de campo.

INDICADORES DE COMPOSICIÓN Y ESTRUCTURA DE LAS TURBERAS OMBROGÉNICAS

Los valores de riqueza total y riqueza promedio de especies de plantas reportados en este estudio, son similares a los valores reportados en la literatura local. Por ejemplo, Henríquez (2004) reportó riquezas totales de turberas esfagnosas de Magallanes, fluctuando en un rango de 14 especies vasculares (San Juan y Parrillar, península de
Brunswick) a 26 especies en Río Pinto (Laguna Blanca). Por otra parte, Domínguez et al. (2012) reportaron un total de 12 especies de plantas vasculares para turberas de Sphagnum no intervenidas de Río Rubens (Última Esperanza). Con respecto a los valores promedio de especies de plantas para turberas ombrogénicas, Domínguez et al. (2012) reportaron $5,23( \pm 0,3)$ especies para censos de $1 \mathrm{~m}^{2}(n=12)$ en turberas de Sphagnum no intervenidas en el sector de Río Rubens (Última Esperanza). Este valor es muy semejante al reportado en el presente estudio $(5,9 \pm 1,3)$ con la misma superficie de muestreo $(n=16)$. Finalmente, los valores promedios reportados por San Martín et al. (1999) para turberas pulvinadas de Donatia en Cordillera Pelada (Valdivia) (8,3 \pm 0,6 , censos de $4 \mathrm{~m}^{2}$ ), son también semejantes a los reportados en el presente estudio $(7,5 \pm 2,0)$. Los bajos valores de riquezas de plantas vasculares reportados para turberas ombrogénicas en Chile han sido extensamente discutidos en la literatura local y están relacionados con condiciones abióticas extremas del hábitat (e.g. baja disponibilidad de nutrientes, anoxia, bajas temperaturas y alta acidez) (Van Breemen 1995, San Martín et al. 1999, Ramírez \& San Martín 2008), y también por propiedades autogénicas del musgo Sphagnum. Van Breemen (1995), por ejemplo, destacó la capacidad del género Sphagnum que, como ingeniero ecosistémico, construye condiciones adversas para la presencia de plantas vasculares en turberas ombrogénicas. Entre los muchos atributos morfológicos, anatómicos, fisiológicos y organoquímicos del género, puede destacarse las paredes celulares del musgo constituidas principalmente de polisacáridos, que le dan una alta capacidad de intercambio catiónico, propiedad responsable de su carácter acídico (Van Breemen 1995). Su naturaleza refractaria, también ayuda a una baja descomposición, que junto a sus paredes de polisacáridos (polímeros polifenólicos) y superficie lipídica, le otorgan un carácter altamente recalcitrante a su hojarasca que impide su descomposición. Esta situación facilita un permanente anegamiento, imposibilitando el ingreso de otras plantas vasculares por anoxia (Van Breemen 1995). Otro aspecto autogénico de relevancia, es la capacidad de Sphagnum de liberar organoquímicos tóxicos que promueven efectos alelopáticos, principalmente ácidos fenólicos que inhiben el crecimiento de otras plantas (Verhoeven \& Liefveld 1997). Esta situación también es extrapolable a las turberas camefíticas con dominancia de Empetrum descritas en este estudio. Empetrum ha sido descrita como una especie cuya hojarasca acidifica el suelo, y una vez descompuesta, libera compuestos tóxicos que finalmente, bloquean la mineralización del nitrógeno, limitando la disponibilidad de nutrientes (Collantes et al. 1989). Estas condiciones limitan el establecimiento de otras plantas vasculares, lo que explica 
en general, la relativamente baja riqueza de especies en las turberas ombrogénicas. Por otra parte, las turberas pulvinadas registraron los valores más altos para la riqueza promedio de especies. Este tipo de turberas se ubicó en el lado negativo del segundo eje de la ordenación, correlacionado con variables de descomposición, lo cual sugiere una mayor disponibilidad de nutrientes en estos sitios. Este patrón de mayor riqueza de especies en sitios más ricos en nutrientes, ha sido observado en turberas ombrotróficas y minerotróficas dominadas por Sphagnum en los alpes italianos y en Suecia (Bragazza \& Gerdol 2002, Bragazza et al. 2005). Variables nutricionales como Calcio, Potasio, Manganeso, Nitrato, Sílice y $\mathrm{N}$ total en mayores concentraciones tanto en agua de poro (pore-water), como en el sustrato turboso, se correlacionaron con los sitios más ricos en especies (Bragazza \& Gerdol 2002). También Vitt \& Chee (1990) describen este patrón para turberas minerotróficas de Alberta, donde las turberas moderadamente ricas en nutrientes y las turberas extremadamente ricas en nutrientes concentran las mayores riquezas de especies. Ambos trabajos dan cuenta de la importancia del gradiente acidez-alcalinidad para las especies de plantas no vasculares y líquenes, mientras que las plantas vasculares son más sensibles al gradiente nutricional del sustrato y del agua de poros (Vitt \& Chee 1990, Bragazza \& Gerdol 2002). En nuestro estudio, la riqueza de no vasculares y líquenes no mostró diferencias significativas entre los distintos tipos de turberas, probablemente al reducido rango de $\mathrm{pH}$ y por cierto, a que nuestro estudio no las determinó a nivel específico. Las plantas vasculares en tanto, mostraron diferencias significativas entre los diferentes tipos de turberas, lo que más probablemente estaría relacionado al gradiente de fertilidad (vinculado a la descomposición del sustrato). Sin embargo, al no contar con variables nutricionales, esta aseveración es de carácter especulativo y requiere ser confirmada.

\section{ESTRUCTURA DE LA VEGETACIÓN DE TURBERAS OMBROGÉNICAS}

Los patrones de dominancia de formas de vida muestran diferencias importantes en el espectro biológico para los diferentes tipos de turberas descritos en el presente estudio. Por una parte, la forma de vida camefítica domina ampliamente el espectro de las turberas esfagnosas, donde la cobertura de Sphagnum magellanicum es mayor. Seguidamente, tanto las turberas camefíticas y pulvinadas son dominadas por los caméfitos, representados esencialmente por especies como Empetrum rubrum y Donatia fascicularis. Finalmente, las turberas graminoides son ampliamente dominadas por criptófitos, donde la especie Marsippospermum grandiflorum es la que representa los mayores valores de cobertura. Esta especie presenta rizomas lineares-oblicuos que sobreviven mientras la parte aérea de la planta tiene tallos efímeros
(Balslev 1998). La característica pulviniforme y esfagnosa de las turberas camefíticas y esfagnosas, facilitarían un anegamiento constante, especialmente por el agua intersticial que acumulan las células hialinas del musgo Sphagnum (Van Breemen 1995), y el agua de lluvia atrapada por las plantas pulviniformes agrupadas en densos cojines, como Donatia fascicularis (Ramírez \& San Martín 2008). Adicionalmente, diversos estudios han mostrado que los atributos morfológicos y anatómicos de musgos del género Sphagnum le proporcionan la propiedad de un mayor aislamiento térmico y mayor recalcitrancia, es decir, una resistencia a la pérdida de masa y de hojarasca (Van Breemen 1995, Dorrepaal et al. 2005, Lang et al. 2009), en contraposición a otras formas de vida como las herbáceas, graminoides y especies caducifolias. Esta condición permitiría, por una parte, una mayor estabilidad ecológica a las turberas dominadas por Sphagnum, especialmente en latitudes extremas y condiciones ambientales desfavorables; como las de la región Magallánica. Por otra parte, las turberas camefíticas y graminoides podrían representar un fenómeno sinérgico de terrestrialización a través de una menor permeabilidad del sustrato y menor aislamiento térmico, que favorecería el futuro establecimiento de nuevas especies ericoides y arbóreas. La transición entre turberas pulvinadas y esfagnosas hacia camefíticas y graminoides, podría representar un gradiente de paludificación a terrestrialización, donde, en este último caso, las raíces de plantas leñosas pueden proporcionar una mayor aireación al sustrato (Bragazza \& Gerdol 2002). Esta condición permitiría el establecimiento y una mayor abundancia de fanerófitos como Nothofagus betuloides.

Perspectivas en inVestigación y Manejo de turberas ombrogénicas PARA RESTAURACIÓN ECOLÓGICA

Las turberas ombrogénicas en Magallanes han sido relativamente bien estudiadas desde el punto de vista fitogeográfico y fitosociológico (e.g. Pisano 1973, 1977, Dollenz 1980, Henríquez 2004, Domínguez et al. 2012), donde se describen principalmente atributos de la vegetación dominante. Consecuentemente, existe información detallada de la flora para estos ecosistemas a una escala geográfica regional. Sin embargo, existe la necesidad de ampliar la escala geográfica de estos estudios, para describir patrones de diversidad a través de gradientes ecológicos de macrositio (e.g. precipitación, temperatura). En este sentido, uno de los pocos estudios realizados a escala geográfica fue el elaborado por Kleinebecker et al. (2007) quienes describieron una cenoclina florística relacionada a un gradiente de continentalidad incrementada, asociada a gradientes de descomposición, profundidad de sustrato y acidez. Estudios de este tipo se hacen importantes para profundizar en gradientes ecológicos 
vinculados a productividad (e.g. biomasa) y almacenamiento de carbono. Por ejemplo, Mathijssen et al. (2019) demostraron que las comunidades de plantas controlan la variabilidad en la acumulación de carbono dentro de una turbera, donde las turberas pulvinadas se descomponen más rápidamente que las turberas de Sphagnum, y en consecuencia acumulan una menor cantidad de Carbono. En este sentido, las turberas dominadas por Sphagnum son muy relevantes como reservorios de carbono, especialmente a la luz de cambios locales como extracción de turba (Domínguez et al. 2012) e impactos globales como cambio climático (Leifeld \& Menichetti 2018). Los principios de restauración ecológica proponen, en términos generales, que los ecosistemas bajo manejo o explotación pueden ser restaurados teniendo una clara referencia sobre los ecosistemas no perturbados (SER 2004). Así, indicadores de biodiversidad estructural, composicional y funcional (sensu Noss 1990) para monitoreo de actividades de manejo y explotación del recurso turba, deben ser elaborados e incorporados en estrategias de manejo ambiental para reiniciar procesos de sucesión en turberas actual o potencialmente intervenidas. Domínguez et al. (2012), por ejemplo, mostraron una incipiente invasión biológica en turberas abandonadas post-extracción minera en turberas del sector del río Rubens (provincia de Última Esperanza, Magallanes), que podría conducir a un estado alternativo al ecosistema original. Así, el diseño de acciones de restauración ecológica activa (e.g. control de especies exóticas), podría ser añadido a planes de manejo en turberas bajo explotación minera. Si bien el presente estudio da cuenta de atributos de composición y estructura de la vegetación de turberas para restauración ecológica a una escala local, estudios a escala regional (e.g. latitudinal), que además incorporen indicadores de funciones ecosistémicas, son altamente requeridos.

\section{AGRADECIMIENTOS}

A Piero Gecele, Miguel Barros y Juan Carlos Vera (Deitan Solutions Spa.) por proporcionar la logística de terreno. Esta investigación fue financiada por Corfo Prototipo de Innovación Regional (17PIRE-72265).

\section{REFERENCIAS}

Amigo, J., San Martín, C., Ramírez, C., Álvarez, M. 2017. Nomenclatural revision and syntaxonomical proposal for wetland peat vegetation in the Valdivian-Magellanian region. Lazaroa 38(2): 165-187.

Balslev, H. 1998. Juncaceae. In: Flowering Plants Monocotyledons. pp. 252-260. Springer, Berlin, Heidelberg.

Bonarelli, G. 1917. Tierra del Fuego y sus turberas. Anales del Ministerio de Agricultura de la Nación, sección Geología, Mineralogía y Minería, Buenos Aires. 120 pp.

Bragazza, L., Gerdol, R. 2002. Are nutrient availability and acidityalkalinity gradients related in Sphagnum-dominated peatlands? Journal of Vegetation Science 13(4): 473-482.

Bragazza, L., Rydin, H., Gerdol, R. 2005. Multiple gradients in mire vegetation: a comparison of a Swedish and an Italian bog. Plant Ecology 177(2): 223-236.

Bubier, J.L., Moore, T.R., Bledzki, L.A. 2007. Effects of nutrient addition on vegetation and carbon cycling in an ombrotrophic bog. Global Change Biology 13(6): 11681186.

Cabrera, A.L., Willink, A. 1973. Biogeografía de América latina. $120 \mathrm{pp}$.

Chen, D., Stow, D. 2002. The effect of training strategies on supervised classification at different spatial resolutions. Photogrammetric Engineering and Remote Sensing, 68(11): 1155-1162.

Chuvieco, E. 2008. Teledetección ambiental, la observación de la tierra desde el espacio. Editorial Ariel, Barcelona, España.

Clarke, K.R. 1993. Non-parametric multivariate analyses of change in community structure. Australian Journal of Ecology 18: 117-143.

Clarke, K.R., Ainsworth, M. 1993. A method of linking multivariate community structure to environmental variables. Marine Ecology Progress Series 92: 205-219.

Clarkson, B.R., Schipper, L.A., Clarkson, B.D. 2004. Vegetation and peat characteristics of restiad bogs on Chatham Island (Rekohu), New Zealand. New Zealand Journal of Botany 42(2): 293-312.

Clutterbuck, B., Chico, G., Labadz, J., Midgley, N.G., 2018. The potential of geospatial technology for monitoring peatland environments. In: Fernández-García, J.M. \& Pérez, F.J. (Eds.) Inventory, Value and Restoration of Peatlands and Mires. pp. 167-181. Recent Contributions, HAZI foundation, Bizkaia.

Clymo, R.S. 1983. Peat. En: Gore, A.J.P. (Eds.) Ecosystems of the world 4A Mires: swamp, bog, fen and moor. General studies. Ecosystems of the world. pp. 159-224. Elsevier Scientific Co. Amsterdam, The Netherlands.

Collantes, M.B., Anchorena, J., Koremblit, G. 1989. A soil nutrient gradient in Magellanic Empetrum heathlands. Vegetatio 80(2): 183-193.

Cottam, G., Curtis, J.T. 1956. The use of distance measures in phytosociological sampling. Ecology 37(3): 451-460.

De Cáceres, M., Legendre, P., Moretti, M. 2010. Improving indicator species analysis by combining group of sites. Oikos 119: 1674-1684. 
De Vleeschouwer, F., Chambers, F.M., Swindles, G.T. 2010. Coring and sub-sampling of peatlands for paleoenvironmental research. Mires and Peat 7(1): 1-10.

Dollenz O. 1980. Estudios fitosociológicos en el archipiélago de Cabo de Hornos. Anales del Instituto de la Patagonia 1(1): 225-238.

Dollenz O. 1982: Estudios fitosociológicos en las Reservas Forestales Alacalufes e Isla Riesco. Anales del Instituto de la Patagonia 13: 161-169.

Domínguez, E. 2012. Flora Nativa: Torres del Paine. Ocho Libros Editores, Santiago. 343 pp.

Domínguez, E. 2014. Manual de buenas prácticas para el uso sostenido del musgo Sphagnum magellanicum en Magallanes, Chile. Instituto de Investigaciones Agropecuarias. Centro Regional de Investigación Kampenaike. Boletín INIA (276). 113 pp.

Domínguez, E., Bahamonde, N., Muñoz-Escobar, A. 2012. Efectos de la extracción de turba sobre la composición y estructura de una turbera de Sphagnum explotada y abandonada hace 20 años, Chile. Anales del Instituto de la Patagonia 40 (2): 37-45.

Domínguez, E., Vega-Valdéz, D. 2015. Funciones y servicios ecosistémicos de las turberas en Magallanes. Colección de libros INIA $\mathrm{N}^{\circ}$ 33. Instituto de Investigaciones Agropecuarias. Centro Regional de Investigación Kampenaike, Punta Arenas, Chile. 334 pp.

Dorrepaal, E., Cornelissen, J.H., Aerts, R., Wallen, B.O., Van Logtestijn, R.S. 2005. Are growth forms consistent predictors of leaf litter quality and decomposability across peatlands along a latitudinal gradient? Journal of Ecology 93(4): 817-828.

Foody, G.M. 2005. Local characterization of thematic classification accuracy through spatially constrained confusion matrices. International Journal of Remote Sensing 26(6): 1217-1228.

Harris, A., Charnock, R., Lucas, R. 2015. Hyperspectral remote sensing of peatland floristic gradients. Remote Sensing of Environment 162: 99-111.

Henríquez, J.M. 2004. Estado de la turba esfagnosa en Magallanes. En: Blanco, D., De la Balze, V. (Eds.), Los turbales de la Patagonia. Bases para su inventario y conservación de la biodiversidad, pp. 93-106. Wetlands International, publicación $\mathrm{N}^{\circ} 19$, Buenos Aires.

Joosten, H., Clarke, D. 2002. Wise use of mires and peatlands. International Mire Conservation Group and International Peat Society, Saarijärvi. 304 pp.

Kimmel, K., Mander, Ü. 2010. Ecosystem services of peatlands: Implications for restoration. Progress in Physical Geography 34(4): 491-514.

Kleinebecker, T., Hölzel, N., Vogel, A. 2007. Gradients of continentality and moisture in South Patagonian ombrotrophic peatland vegetation. Folia Geobotanica 42(4): 363-382.

Lang, S.I., Cornelissen, J.H., Klahn, T., Van Logtestijn, R.S., Broekman, R., Schweikert, W., Aerts, R. 2009. An experimental comparison of chemical traits and litter decomposition rates in a diverse range of subarctic bryophyte, lichen and vascular plant species. Journal of Ecology 97(5): 886-900.

Larraín, J. 2007. Musgos (Bryophyta) de la estación biológica Senda Darwin, Ancud, isla de Chiloé: lista de especies y claves para su identificación. Chloris Chilensis 10(1). URL: http://www.chlorischile.cl. Accedido: Marzo 29, 2019.

Leifeld, J., Menichetti, L. 2018. The underappreciated potential of peatlands in global climate change mitigation strategies. Nature communications 9(1): 1071.

Luebert, F., Pliscoff, P. 2006. Sinopsis bioclimática y vegetacional de Chile. Editorial Universitaria, Santiago.

Mathijssen, P.J.S., Galka., M., Borken, W., Holger-Knorr, K. 2019. Plant communities control long term carbon accumulation and biogeochemical gradients in a Patagonian bog. Science of the Total Environment 684: 670-681.

Miao, X., Patil, R., Heaton, J.S., Tracy, R.C. 2011. Detection and classification of invasive saltcedar through high spatial resolution airborne hyperspectral imagery. International Journal of Remote Sensing 32(8): 2131-2150.

MINAGRI. 2018. Decreto 25. Dispone medidas para la protección del musgo Sphagnum magellanicum. Ministerio de Agricultura.

Moore, D.M. 1983. Flora of Tierra del Fuego. Anthony Nelson, Oswestry, England. 396 pp.

Mueller-Dombois, D., Ellenberg, H. 1974. Aims and methods of vegetation ecology. John Wiley \& Sons, New York, USA.

Noss, R.F. 1990. Indicators for monitoring biodiversity: a hierarchical approach. Conservation Biology 4(4): 355364.

Oberdorfer, E. 1960. Pflanzensoziologische studien in Chile: Ein Vergleich mit Europa. J. Cramer.

Pérez-Haase, A., Iturraspe, R., Ninoti, J.M. 2019. Macroclimate and local hydrological regime as drivers of fen vegetation patterns in Tierra del Fuego (Argentina). Ecohydrology 12(8). doi: 10.1002/eco.2155

Pisano, E. 1973. Fitogeografía de la Península de Brunswick Magallanes. I. Comunidades Meso-hidromórficas. Anales del Instituto de la Patagonia 4 (1-3): 141-206.

Pisano, E. 1977. Fitogeografía de Fuego - Patagonia Chilena. I. Comunidades vegetales entre las latitudes $52^{\circ}$ y $56^{\circ} \mathrm{S}$. Anales del Instituto de la Patagonia 8: 121-250.

Ramírez, C., San Martín, C. 2008. Ecosistemas dulceacuícolas. En: CONAMA (Eds.), Biodiversidad de Chile: Patrimonio 
y Desafíos. pp. 112-124. Ocho Libros Editores, Santiago, Chile.

Rodríguez, R., Marticorena, C., Alarcón, D., Baeza, C., Lohengrin, C., Finot, V., Fuentes, N., Kiessling, A., Mihoc, M., Pauchard, A., Ruiz, E., Sánchez, P., Marticorena, A. 2018. Catálogo de las plantas vasculares de Chile. Gayana Botánica 75(1): 1-430.

Ruiz, J., Doberti, M. 2005. Catastro y Caracterización de los Turbales de Magallanes. Punta Arenas, Chile.

San Martín, C., Ramírez, C., Figueroa, H. 1999. Análisis multivariable de la vegetación de un complejo de turberas en Cordillera Pelada (Valdivia, Chile). Lazaroa 20: 95-106.

SER. 2004. The SER International Primer on Ecological Restoration. Society for Ecological Restoration International Science and Policy Working Group. Society for Ecological restoration International, Tucson, USA.

SERNAGEOMIN. 2018. Anuario de la minería de Chile, 2017. Servicio Nacional de Geología y Minería de Chile, Chile.

Sokal, R.R., Rohlf, F.J. 1980. Comments on taxonomic congruence. Systematic Zoology 29(1): 97-101.

Succow, M., Joosten, H. 2001. Landschaftsökologische Moorkunde. 2nd ed. Stuttgart: Schweizerbart. 622 pp.

Tapia, M. 2008. Crecimiento y productividad del musgo Sphagnum magellanicum Brid. turberas secundarias de la provincia de Llanquihue, Chile. Tesis de Agronomía. Facultad de Ciencias Agrarias, Universidad Austral de Chile, Valdivia,
Chile. 74 pp.

Tobler, W. 1987. Measuring spatial resolution. In: Proceedings of the International Workshop on Geographic Information Systems (Vol. 48). pp. 12-16. International Geographic Union, Beijing.

Van Breemen, N. 1995. How Sphagnum bogs down other plants. Trends in Ecology \& Evolution 10(7): 270-275.

Verhoeven, J.T.A., Liefveld, W.M. 1997. The ecological significance of organochemical compounds in Sphagnum. Acta Botanica Neerlandica 46(2): 117-130.

Vidal, O. 2006. Flora Torres del Paine: guía de campo. Fantastico Sur, Punta Arenas. 283 pp.

Vitt, D.H., Chee, W.L. 1990. The relationships of vegetation to surface water chemistry and peat chemistry in fens of Alberta, Canada. Vegetatio 89(2): 87-106.

Von Post, L. 1924. Das genetische System der organogenen Bildungen Schwedens. IVème commission pour l'Europe. Comité international de Pédologie.

Wheeler, B.D., Proctor, M.C.F. 2000. Ecological gradients, subdivisions and terminology of north-west European mires. Journal of Ecology 88(2): 187-203.

Yonezawa, C. 2007. Maximum likelihood classification combined with spectral angle mapper algorithm for high resolution satellite imagery. International Journal of Remote Sensing 28(16): 3729-3737.

Received: 27.05.2019

Accepted: 03.12.2020 
AneXo 1. Catálogo de especies de plantas vasculares y no vasculares registradas en los censos fitosociológicos realizados para el presente estudio, con valores de frecuencia absoluta (F.ab.), cobertura absoluta (C.ab) y valor de importancia (V.I.) para cada tipo de vegetación en turberas ombrogénicas. / Catalog of vascular and non-vascular plant species recorded in the phytosociological surveys carried out for the present study, indicating values of absolute frequency (F.ab.), absolute cover (C.ab) and importance value (V.I.) for each vegetation type of ombrogenic bogs.

Especies

Turbera graminoide Turbera camefítica Turbera esfagnosa Turbera pulvinada

\begin{tabular}{|c|c|c|c|c|c|c|c|c|c|c|c|c|}
\hline & F.ab & C.ab & V.I & F.ab & C. $a b$ & V.I & F.ab & C.ab & V.I & F.ab & C. $a b$ & V.I \\
\hline Avenella flexuosa (L.) Drejer & 0 & 0 & 4,3 & 1 & 20 & 2,9 & 0 & 0 & 0,0 & 0 & 0 & 0,0 \\
\hline Aulacomnium palustre (Hedw.) Schwägr. & 0 & 0 & 0,0 & 1 & 1 & 1,5 & 0 & 0 & 0,0 & 0 & 0 & 0,0 \\
\hline Berberis microphylla G.Forster & 0 & 0 & 0,0 & 0 & 0 & 0,0 & 0 & 0 & 0,0 & 0 & 0 & 0,0 \\
\hline Blechnum penna-marina (Poir.) Kuhn & 1 & 1 & 2,3 & 0 & 0 & 0,0 & 0 & 0 & 0,0 & 0 & 0 & 0,0 \\
\hline Caltha appendiculata Pers. & 0 & 0 & 0,0 & 1 & 2 & 1,6 & 0 & 0 & 0,0 & 2 & 2 & 7,3 \\
\hline Carex magellanica Lam. & 0 & 0 & 55,3 & 0 & 0 & 0,0 & 2 & 26 & 3,9 & 0 & 0 & 0,0 \\
\hline Chiliotrichum diffusum (G. Forst.) Kuntze & 1 & 10 & 0,0 & 0 & 0 & 0,0 & 0 & 0 & 0,0 & 0 & 0 & 0,0 \\
\hline Cladonia spp. & 2 & 16 & 48,5 & 8 & 148 & 22,1 & 6 & 94 & 12,9 & 3 & 26 & 17,6 \\
\hline Dicranoloma dusenii (Broth.) Broth. & 1 & 2 & 18,7 & 0 & 0 & 0,0 & 0 & 0 & 0,0 & 0 & 0 & 0,0 \\
\hline Donatia fascicularis J.R. Forst. \& G. Forst. & 0 & 0 & 9,1 & 0 & 0 & 0,0 & 0 & 0 & 0,0 & 4 & 195 & 70,4 \\
\hline Drosera uniflora Willd. & 1 & 1 & 0,0 & 1 & 5 & 1,8 & 8 & 42 & 11,3 & 2 & 7 & 8,7 \\
\hline Empetrum rubrum Vahl ex Willd. & 6 & 190 & 0,0 & 15 & 829 & 81,5 & 16 & 262 & 35,1 & 3 & 37 & 20,8 \\
\hline Gaultheria antarctica Hook.f. & 4 & 23 & 0,0 & 13 & 47 & 21,7 & 15 & 43 & 18,8 & 3 & 3 & 10,9 \\
\hline Gaultheria mucronata (L.f.) Hook. \& Arn. & 1 & 10 & 0,0 & 1 & 15 & 2,5 & 0 & 0 & 0,0 & 0 & 0 & 0,0 \\
\hline Gaultheria pumila (L.f.) D.J. Middleton & 0 & 0 & 3,5 & 3 & 12 & 5,1 & 1 & 3 & 1,3 & 0 & 0 & 0,0 \\
\hline Gunnera magellanica Lam. & 1 & 10 & 0,0 & 0 & 0 & 0,0 & 0 & 0 & 0,0 & 0 & 0 & 0,0 \\
\hline Marsippospermum grandiflorum (L.f.) Hook. f. & 7 & 285 & 0,0 & 3 & 15 & 5,3 & 1 & 2 & 1,2 & 0 & 0 & 0,0 \\
\hline Misodendrum punctulatum DC. & 1 & 5 & 0,0 & 0 & 0 & 0,0 & 0 & 0 & 0,0 & 0 & 0 & 0,0 \\
\hline Myrteola nummularia (Poir.) O. Berg & 0 & 0 & 2,2 & 1 & 5 & 1,8 & 0 & 0 & 0,0 & 2 & 6 & 8,4 \\
\hline Nanodea muscosa Banks ex C.F.Gaertn. & 1 & 1 & 2,2 & 3 & 4 & 4,5 & 4 & 5 & 4,6 & 0 & 0 & 0,0 \\
\hline Nothofagus antarctica (G. Forst.) Oerst. & 1 & 2 & 2,2 & 3 & 8 & 4,8 & 7 & 14 & 8,3 & 1 & 1 & 3,6 \\
\hline Nothofagus betuloides (Mirb.) Oerst. & 1 & 70 & 2,2 & 3 & 8 & 4,8 & 6 & 7 & 6,8 & 3 & 4 & 11,2 \\
\hline Oreobolus obtusangulus Gaudich. & 0 & 0 & 3,5 & 0 & 0 & 0,0 & 0 & 0 & 0,0 & 1 & 2 & 3,9 \\
\hline Polypogon magellanicus (Lam.) Finot & 1 & 1 & 0,0 & 0 & 0 & 0,0 & 0 & 0 & 0,0 & 0 & 0 & 0,0 \\
\hline Pseudocifelaria spp. & 0 & 0 & 5,4 & 1 & 3 & 1,6 & 0 & 0 & 0,0 & 0 & 0 & 0,0 \\
\hline Senecio acanthifolius Hombr. \& Jacquinot & 0 & 0 & 2,8 & 0 & 0 & 0,0 & 0 & 0 & 0,0 & 0 & 0 & 0,0 \\
\hline Sphagnum falcatulum Besch. & 0 & 0 & 4,6 & 0 & 0 & 0,0 & 0 & 0 & 0,0 & 0 & 0 & 0,0 \\
\hline Sphagnum fimbriatum Wilson. & 0 & 0 & 4,6 & 0 & 0 & 0,0 & 0 & 0 & 0,0 & 0 & 0 & 0,0 \\
\hline Sphagnum magellanicum Brid. & 4 & 59 & 11,8 & 9 & 236 & 29,9 & 16 & 845 & 75,6 & 3 & 41 & 22,0 \\
\hline Tetroncium magellanicum Willd. & 0 & 0 & 2,2 & 4 & 14 & 6,7 & 13 & 95 & 20,3 & 3 & 18 & 15,3 \\
\hline Usnea spp. & 0 & 0 & 15,0 & 0 & 0 & 0,0 & 0 & 0 & 0,0 & 0 & 0 & 0,0 \\
\hline
\end{tabular}

